\title{
Multiple urinary bladder calculi: a rare cause of irreducible uterovaginal prolapse
}

\author{
Manjit Kaur Mohi, Manjeet Kaur, Balwinder Kaur, Satinder Pal Kaur, Sangeeta Aggarwal*
}

Department of Obstetrics and Gynecology, GMC, Patiala, Punjab, India

Received: 06 October 2021

Revised: 10 November 2021

Accepted: 11 November 2021

\section{*Correspondence:}

Dr. Sangeeta Aggarwal,

E-mail: drsangeetaaggarwal@gmail.com

Copyright: (c) the author(s), publisher and licensee Medip Academy. This is an open-access article distributed under the terms of the Creative Commons Attribution Non-Commercial License, which permits unrestricted non-commercial use, distribution, and reproduction in any medium, provided the original work is properly cited.

\begin{abstract}
A case of irreducible prolapse with multiple bladder calculi in a 65-years-old multiparous, postmenopausal woman is reported. Inability to walk, constipation and urinary incontinence were her primary complaints. Routine ultrasound of the abdomen and pelvis failed to reveal multiple vesical calculi as the prolapse was lying outside the pelvis. However, targeted plain X-ray of the prolapsed mass showed multiple vesical calculi. The patient was managed with single-stage vaginal hysterectomy and laparotomy. First vaginal hysterectomy was done then prolapsed cystocele was reduced and extraperitoneal vesicolithotomy done. Currently, the patient is relieved of all symptoms. Management of an irreducible procidentia and a hard mass in the anterior compartment, as in this case, can be challenging and requires a diligent effort to confirm the diagnosis and to execute the appropriate surgical protocol to achieve optimal outcome with minimal intra- and post-operative complications.
\end{abstract}

Keywords: Procidentia, Vesical calculi, Irreducible

\section{INTRODUCTION}

Irreducibility of uterovaginal prolapse can occur due to uterine, bladder or bowel pathology. Uterine fibroid or large bladder calculi are the common causes of irreducibility. ${ }^{1}$ Rarely, an oedematous, hypertrophied, congested cervix with normal uterus can cause irreducibility. Here we reported a case of long-standing, neglected irreducible prolapse owing to large multiple bladder calculi.

\section{CASE REPORT}

A 65 years old multiparous (para 3) female, 15 years postmenopausal, presented with a mass coming out per vagina for the last 20 years. The prolapse has increased in size gradually. Earlier, she used to reduce the prolapse digitally in order to evacuate the bladder. During the last 6 months, there has been significant restriction of physical activity because of increase in size and irreducibility. She was brought to gynecology department of Rajindra hospital,
Patiala as an emergency patient because of irreducibility of prolapse and severe restriction of ambulation. Her urinary symptoms included increased frequency (10-15 times per day, 4-5 times per night), burning micturition, urgency and severe urge incontinence. Constipation (once in 6-7 days) and was an associated symptom. There was no comorbidity like diabetes mellitus or hypertension.

On clinical examination, the patient was short, thin and undernourished $\left(\mathrm{BMI}=18 \mathrm{~kg} / \mathrm{m}^{2}\right)$. The rest of the general and systemic examination findings were within normal limits. Local examination revealed a huge prolapse $(15 \times 10 \times 10 \mathrm{~cm})$ with large cystocele and enterocele (Figure 1A). There was no cervical hypertrophy or congestion and cervical OS was identified with difficulty. The vaginal wall was thickened and the prolapse was irreducible. Multiple ulcers were present on anterior vaginal wall. Multiple hard masses were palpable through it. Rectal examination was normal. According to POPQ classification there was stage IV Pelvic organ prolapse (POP) involving all the compartments and complete 
eventration of the vagina with ' $C$ ' point at +15 . Patient was catheterised and managed by bed rest, elevation of foot end of bed, local application of glycerine, acriflavin and estrogen cream in order to reduce the oedema and heal the ulcer.

Renal function tests were deranged. Ultrasound of the pelvic organs was suggestive of bilateral hydroureteronephrosis grade II to III. Plane X-ray pelvic organs showed multiple stones in prolapsed part of urinary bladder (Figure 1B). MRI of the abdomen and pelvic organs confirmed multiple stones in bladder, prolapsed ureterovesical junction and obstructive uropathy.

The patient was electively taken up for surgery. Cervical OS was identified. Incision given anterior to OS on vaginal wall. Cystocele dissected away from cervix and uterus. Vaginal hysterectomy was performed. The hysterectomy facilitated reduction of the remaining prolapse. The urologist was summoned intraoperatively for further management as the mass was identified to be mutiple vesical calculi.

The urologist performed a suprapubic extraperitoneal vesicolithotomy. Incision of the thickened, oedematous anterior wall of the bladder exposed its cavity filled with foul-smelling calculi. Complete removal of the bladder calculi (9 in number) varying in size, from 1 to $6 \mathrm{~cm}$ in diameter was done (Figure 2A). The bladder was closed in layers, retaining a suprapubic catheter and urethral catheter. Vaginal vault closure was carried out after excision of the redundant vaginal wall and pelvic floor repair was done (Figure $2 \mathrm{~B}$ ).

Post-operatively two units of blood transfusion along with parenteral higher antibiotics were given. She required intense monitoring postoperatively. Suprapubic catheter was removed after 72 hours. Continuous bladder drainage was maintained for 4 weeks. The patient was discharged with normal renal parameters and sterile urine. She is symptom-free at 1-month follow-up.

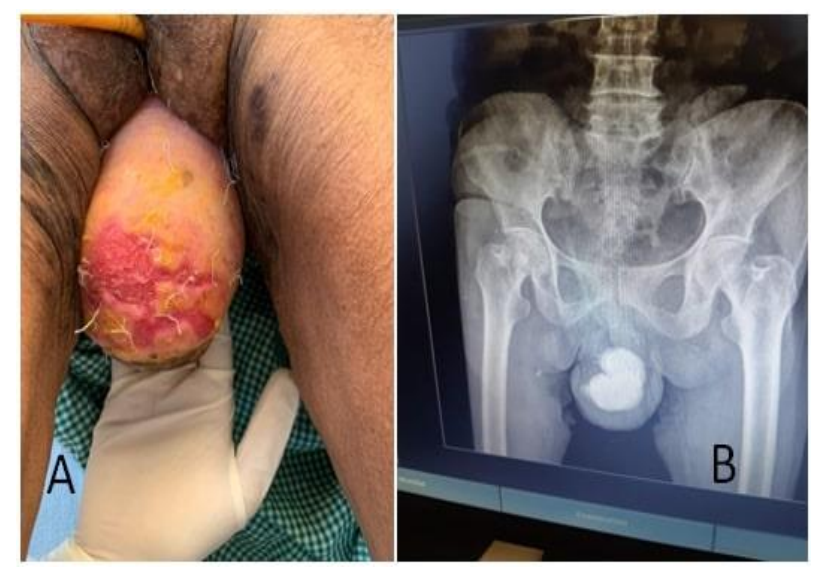

Figure 1: (A) Showing irreducible uterovaginal prolapse; and (B) plain X-ray showing multiple stones in prolapsed part.

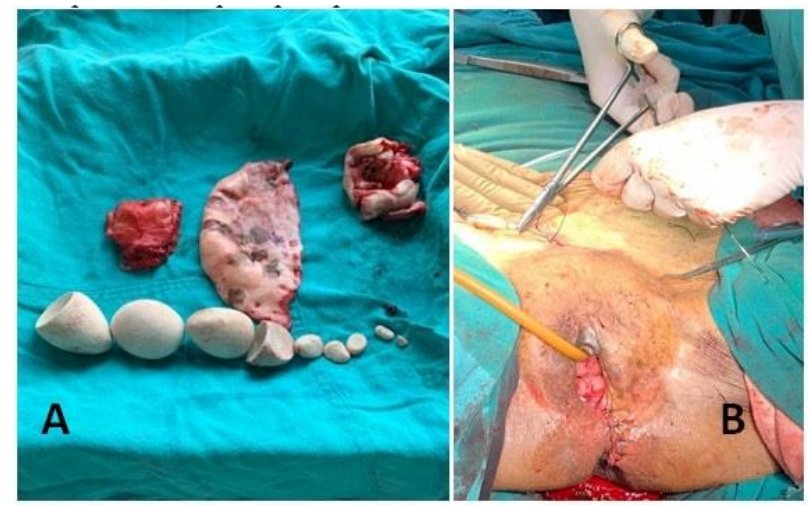

Figure 2: (A) Showing multiple stones removed from bladder; and (B) at the end of surgery.

\section{DISCUSSION}

Large uterine fibroid or bladder calculi can render the prolapse irreducible. Co-existing bladder calculi in cases of procidentia are invariably due to stasis secondary to obstruction of the outflow tract. Vesical calculi in turn can aggravate the obstructive voiding symptoms and exacerbate events. Failure to identify the pathology by USG in this patient was due to the anatomical descent and distortion associated with procidentia.

Management of an irreducible procidentia and a hard mass in the anterior compartment, as in this case, can be challenging and requires a diligent effort to confirm the diagnosis and to execute the appropriate surgical protocol to achieve optimal outcome with minimal intra- and postoperative complications. An irreducible procidentia with a mass in the anterior vaginal wall, the diagnosis of vesical calculi should be considered unless proven otherwise. The role of targeted plain X-ray of the prolapsed mass is invaluable in clinching the diagnosis. The challenges of surgically removing multiple bladder calculi in complete vaginal prolapse were discussed by Wai et al. ${ }^{2}$ Stones may be removed vaginally, suprapubically, using cystoscopic resection, or by extracorporeal shockwave lithotripsy. Wai et al. used a suprapubic approach with cystotomy to facilitate complete removal of multiple small stones.

Nieder et al also advocated suprapubic cystolithotomy as a means for improved evaluation of the bladder and to facilitate ease of stone removal. ${ }^{3}$ Agarwal et al reported a case of irreducible uterine prolapse with large bladder stone. They did suprapubic extraperitoneal cystolithotomy removed the stone and later on did vaginal hystrectomy. Washington et al reported a similar case in which the vaginal hysterectomy was completed, a cystotomy was made in the posterior detrusor muscle away from the ureters. The stones were easily removed under excellent visualization. ${ }^{5}$ Rajeshwari et al has reported a similar case of irreducible prolapse due multiple bladder calculi which was also managed by vaginal hystrectomy and extraperitoneal vesicolithotomy. ${ }^{6}$ 


\section{CONCLUSION}

Bladder calculi can render the prolapse irreducible. Coexisting bladder calculi in cases of procidentia are invariably due to stasis secondary to obstruction of the outflow tract. Vesical calculi in turn can aggravate the obstructive voiding symptoms and exacerbate events. Failure to identify the pathology by USG in these patients is due to the anatomical descent and distortion associated with procidentia. The role of targeted plain X-ray of the prolapsed mass is invaluable in clinching the diagnosis. Management was done by vaginal hystrectomy and suprapubic vesicolithotomy.

Funding: No funding sources

Conflict of interest: None declared

Ethical approval: Not required

\section{REFERENCES}

1. Partoll L, Miklos JR, Karram MM. Uterine procidentia incarcerated by bladder calculus. A case report and review of literature. J Pelvic Surg. 1996;2(2):55-7.

2. Wai CY, Margulis V, Baugh BR, Schaffer JI. Multiple vesical calculi and complete vaginal vault prolapse. Am J Obstet Gynecol. 2003;189(3):884-5.

3. Nieder AM, Chun TY, Nitti VW. Total vaginal prolapse with multiple vesical calculi after hysterectomy. J Urol. 1998;159(3):983.

4. Dalela D, Agarwal R. Large vesical calculus in a cystocoele: an uncommon cause of irreducible genital prolapse. BJU Int. 1999;84(1):171-2.

5. Washington B, Hines B, Stoneburg S. Bladder calculi presenting as complete procidentia. Int Urogynecol $\mathbf{J}$ Pelvic Floor Dysfunct. 2008;19(1):157-9.

6. Rajamaheswari N, Agarwal S, Krishnan S. Irreducible procidentia due to multiple bladder calculi mimicking impacted faecal mass. Int Urogynecol J. 2012;23(10):1475-7.

Cite this article as: Mohi MK, Kaur M, Kaur B, Kaur SP, Aggarwal S. Multiple urinary bladder calculi: a rare cause of irreducible uterovaginal prolapse. Int J Reprod Contracept Obstet Gynecol 2021;10:4593-5. 